\title{
Green approach to Chemo-Selective N-Boc Protection of Amines using Catalytic amount of Lithium Hydroxide Monohydrate under Solvent Free Condition
}

\author{
Sandip P. Gondake* , Santosh R. Kshirsagar, Ashok S. Pise, Valmik S. Kapase, Sagar I. Shinde \\ Department of Chemistry, Dada Patil Mahavidyalaya, Karjat, Ahmednagar, Maharashtra, India \\ *Corresponding author: Email : gondakesandip12@gmail.com
}

\begin{abstract}
Article Info
Volume 7, Issue 5

Page Number: 06-12

Publication Issue :
\end{abstract}

September-October-2020

\section{Article History}

Accepted : 01 Sep 2020

Published : 04 Sep 2020

\section{ABSTRACT}

The protecting group plays important role in synthesis of multifunctional targets. A simple rapid efficient and green method for chemo-selective N-Boc protection of amines using Lithium hydroxide as a green catalyst. In the present work amine protection in presence of ditert-butyl carbonate under solvent free condition is carried out. An efficient green protocol for chemoselctive $\mathrm{N}-\mathrm{Boc}$ protection of aryl, aliphatic, aromatic, acyclic and hetero cyclic amines $(1 \mathrm{~m} \mathrm{~mol} \%)\left(1^{0}, 2^{0}, 3^{0}\right)$ were carried out with ditert-butyl carbonate (Boc) ${ }_{2} \mathrm{O}$ using Lithium hydroxide monohydrate $(10 \mathrm{~mol} \%)$ at room temperature under solvent free condition to give white solid monitored on TLC. No side reactions are observed .The present protocol were simple, rapid, efficient, shorter reaction times, high yielding, highly selective, economical and eco-friendly

Keywords: N-Boc, Lithium hydroxide monohydrate, protection of amines, Solvent free, chemo-selective, green method.

\section{INTRODUCTION}

The environmental friendly and economically inexpensive synthetic procedures are being developed to reduce the harmful effect of organic solvent on environment is major challenge in synthetic and medicinal Chemistry. The amino functional group has important role in biological functions as well as organic synthesis. The protection and de-protection of organic functional groups play vital role in multistep organic synthesis [1]. There is need to protection amine function may biological active compound frequently used in synthetic and medicinal chemistry. Among them, the protection of N-tertbutoxylcarbonylation has great attention due to extra stability of N-Boc group towards nucleophilic attack, alkaline condition and catalytic hydrogenation [2].

The various regent and methods have been used during last few years for the of N-tertbutoxylcarbonylation amines most them are (Boc) 20 in the presence of base catalyzed DMPA [3], 4dimethylamino-1-tert-butoxycarbonyl pyridinium chloride[4], $\mathrm{K}_{2} \mathrm{CO}_{3}$ ( Potassium Carbonate)[5], Et $3 \mathrm{~N}$ ( Tri-ethyl amine) [6]. However these methods has drawback like long reaction time, preparation of 
reagent, requirement of solvents and other reagents.These protocols lead to the formation of side product isocyanate, urea[7]. In addition, high toxicity, unpleasant smell, non-recyclability requirement, excess amount of catalyst makes these methods objectionable, especially from the view of green chemistry.

The acid catalyzed N-Boc protection of amines studied using homogeneous and heterogeneous catalysts. These procedures corrosive and moisture sensitive reagent $\mathrm{ZrClO}_{4}$ [8], $\mathrm{H}_{3} \mathrm{PW}_{12} \mathrm{O}_{14}$ [9], $\mathrm{H}_{2} \mathrm{NSO}_{3} \mathrm{H}$ [10], $\mathrm{FeCl}_{3}[11], \mathrm{I}_{2}$ [12], Thiourea [13], $\mathrm{LiClO}_{4}$ [14], $\mathrm{Zn}\left(\mathrm{ClO}_{4}\right)_{2} .6 \mathrm{H}_{2} \mathrm{O} \quad[15], \mathrm{La}\left(\mathrm{NO}_{3}\right)_{3}[16], \mathrm{Cu}\left(\mathrm{BF}_{4}\right)_{2} .6 \mathrm{H}_{2} \mathrm{O}$ [17], $\mathrm{HClO}_{4}$ [18] ,Functionalized Silica [19], $\mathrm{InCl}_{3}, \mathrm{InBr} 3$ [20], TFE [21], CsF [22], Montmorillonite $\mathrm{K} 10$ [23], $\left(\mathrm{CF}_{3}\right)_{2} \mathrm{CHOH}$ [24], thioglycolurial [25], sulphamic acid[26],guanidine hydrochloride [27], sulfonic acid been used. The heterogeneous catalysts has long and tedious procedures (to prepare the silica sulfonic acid catalyst, 3-4 days are required) [28] or calcinations at high temperature (500C for yttriazirconia) [29]. The most of the N-Boc Protection reactions are slow (1-22 hrs) with electron deficient amines.

The catalyst free protocol include use of cyclodextrin [30], water [31], ethanol [32], and PEG-400 [33], as well as solvent free condition with and without microwave irradiation [34], are used for protection of amines.The protocol has been developed for chemoselective N-Boc protection of amines using a glycerol solvent at room temperature [35], sulphamic acid as a catalyst [36], 1,1,3,3,-tetramethylgauinidinium acetate (10 $\mathrm{mol} \%)$ as a recyclable catalyst under solvent free condition at ambient temperature [37].

But these methods has suffers certain limitations as less yield, toxic catalyst \& solvents, slow reaction rate, tedious workup, recyclability of catalyst and solvent. Thus design of new safe eco-friendly, efficient, high yielding strategies, and solvent free and chief N-Boc protection of amines still is an active topic in synthetic chemistry.

In present work, we have design an efficient green protocol for chemo-selective $\mathrm{N}$-Boc protection of amines using Lithium hydroxide monohydrate as catalyst at room temperature. The reaction between aromatic amine (1mmol), ditert-butyl carbonate (Boc) ${ }_{2} \mathrm{O}(1 \mathrm{~m} \mathrm{~mol})$ in presence of $\mathrm{LiOH} . \mathrm{H}_{2} \mathrm{O}$ stirred at room temperature to give white solid monitored on TLC. In presences of electron rich substituent's very rapid reaction occurred with high yields. While in presences of electron deficient substituent's slow reaction occurs with low yield. The effect of amount of catalyst on yield and performances of various catalysts in the $\mathrm{N}$-Boc protection of amines would be compared. No competitive side reactions are observed in this protocol.

\section{EXPERIMENTAL}

\subsection{Apparatus and Analysis}

The melting points of the compounds were determined are uncorrected. The synthesized products were monitored on silica gel $G$ plates by using pet ether: ethyl acetate (7:3) as mobile phase. FT-IR spectra were recorded on a Shimadzu Miracle 10 ATR spectrometer. ${ }^{1} \mathrm{H}$ NMR spectra were recorded on a Bruker $500 \mathrm{MHz}$ spectrometer with $\mathrm{CDCl}_{3}$ as the solvent and TMS as the internal reference. ${ }^{13} \mathrm{C}$ NMR spectra were recorded on Bruker $125 \mathrm{MHz}$ spectrometer with $\mathrm{CDCl}_{3}$ as the solvent. All compounds are purified by column chromatography using silica gel (70-230 mesh) and solvents pet-ether: ethyl acetate as 7:3. Elemental analysis carried out using CHN elemental analyzer. 


\subsection{General Experimental procedure:}

The reaction between aromatic/aliphatic /acyclic and heterocyclic amine $(1 \mathrm{mmol})$, ditert-butyl carbonate (Boc) ${ }_{2} \mathrm{O}(1 \mathrm{mmol})$ in presence of $\mathrm{LiOH}_{2} \mathrm{H}_{2} \mathrm{O}(2 \mathrm{mmol})$ stirred at room temperature to give white solid formed the progress of reaction is monitored on TLC. After completion of reaction, the reaction mixture was extracted with $5 \mathrm{ml}$ diethyl ether. The organic layer was washed with brine solution and dried over anhydrous sodium sulfate. The crude product was purified by using silica gel column chromatography (30\% ethyl acetate in light petroleum) to get pure white solid product with excellent yield. No competitive side reactions are observed in this protocol.<smiles>Nc1ccccc1</smiles>

1 a

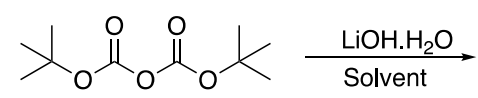

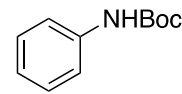

3a
Scheme- I: N-Boc protection catalyzed by Lithium hydroxide monohydrate

\section{RESULTS AND DISCUSSION}

We consciously chose the $\mathrm{LiOH} . \mathrm{H}_{2} \mathrm{O}$ because it is soluble in water, strong base, and easy to remove from reaction mixture by simply water filtration/extraction. We began our investigations for model reaction between aniline $1 \mathbf{a}$ and $(\mathrm{Boc})_{2} \mathrm{O} 2 \mathbf{2}$ as substrates (1:1) at room temperature, and we were encouraged by the good results; formation of N-Boc protected aniline 3a in $98 \%$ yield in $\mathrm{H}_{2} \mathrm{O}$ as solvent after only $2 \mathrm{~min}$. without any side products (Table 1 , entry 1). The model reaction could also be carried out under solvent free condition and obtained excellent results.It is comprehensible from Table 1, most prominently, that $\mathrm{LiOH} . \mathrm{H}_{2} \mathrm{O}$ was found to be the best catalyst in terms of reaction times and yields (97\%, 2 min, entry 2, Table 1), compared with the reported results using LA's or additives (entries 5-7, Table 1).
Table 1. Optimization of the Reaction Conditions $s^{\mathrm{a}, \mathrm{b}}$ and Comparison of the Effect of Catalysts in $N$-Boc Protection of Aniline with (Boc) $)_{2} \mathrm{O}$

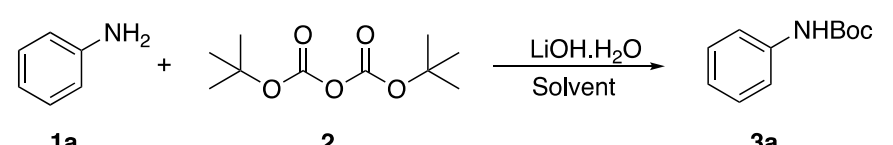

\begin{tabular}{|c|c|c|c|c|c|}
\hline Entry & Solvent & Catalyst & $\begin{array}{l}\text { Catalyst } \\
\text { (mol\%) }\end{array}$ & Time & $\begin{array}{c}\text { 3a } \\
\text { Yield } \\
\text { (\%) }\end{array}$ \\
\hline 1 & $\mathrm{H}_{2} \mathrm{O}$ & $\mathrm{LiOH} . \mathrm{H}_{2} \mathrm{O}$ & 10 & $\begin{array}{c}2 \\
\min \end{array}$ & 98 \\
\hline 2 & - & $\mathrm{LiOH} . \mathrm{H}_{2} \mathrm{O}$ & 10 & $\begin{array}{c}2 \\
\min \end{array}$ & 97 \\
\hline 3 & - & $\mathrm{LiOH} . \mathrm{H}_{2} \mathrm{O}$ & 20 & $\begin{array}{c}2 \\
\min \end{array}$ & 98 \\
\hline 4 & - & $\mathrm{LiOH} . \mathrm{H}_{2} \mathrm{O}$ & 30 & $\begin{array}{c}2 \\
\min \end{array}$ & 98 \\
\hline 5 & $\mathrm{CH}_{3} \mathrm{CN}$ & $\begin{array}{l}\text { ytttria - } \\
\text { zirconia }\end{array}$ & 10 & $14 \mathrm{~h}$ & 90 \\
\hline 6 & $\mathrm{CH}_{2} \mathrm{Cl}_{2}$ & $\mathrm{Zn}\left(\mathrm{ClO}_{4}\right)_{2.6 \mathrm{H}_{2} \mathrm{O}}$ & 5 & $12 \mathrm{~h}$ & 92 \\
\hline 7 & - & $\mathrm{I}_{2}$ & 10 & $\begin{array}{l}30 \\
\min \end{array}$ & 95 \\
\hline
\end{tabular}

aReaction conditions: 1a (1 mmol), 2 (1 mmol), $\mathrm{LiOH} . \mathrm{H}_{2} \mathrm{O}(10 \mathrm{~mol} \%)$, in Solvent $(1 \mathrm{~mL})$ at room temperature for given time. bIsolated.

After optimised reaction condition in hand, we then explored the scope and generality of this reaction with various anilines $1 \mathbf{a}-\mathbf{m}$ and (Boc) ${ }_{2} \mathrm{O} 2 \mathbf{2 a}$ (Table 2). The presence of a substituent on the aryl ring of aniline1 had effect on chemical yields. Using electron donating groups at ortho, meta, and para- substituted aniline 1 , afforded the corresponding products $3 \mathrm{~b}-\mathrm{g}$ in very good yield (91-99\%). But in case of electron withdrawing groups at the meta and para position the yield of corresponding products $3 \mathrm{~h}-\mathrm{i}$ slightly decrease (82-85\%), and time of the reaction also increases. The reaction was found to be sensitive to the disubstituted aniline $\mathbf{3} \mathbf{j}$ reaction proceed in a sluggish manner and give $65 \%$ yield after $25 \mathrm{~min}$. Aniline with para-halogroups the corresponding products $3 \mathrm{k}$ and 31 were obtained in moderate yields $(88 \%$ and $82 \%)$ 
respectively. Additionally, this procedure was also applicable to hetero-aryl amine $3 \mathrm{~m}$ and afforded very good yield (96\%).

Table 2. $\mathrm{LiOH}_{2} \mathrm{H}_{2} \mathrm{O}$-Catalyzed Boc Protection of Amines $^{\mathrm{a}, \mathrm{b}}$

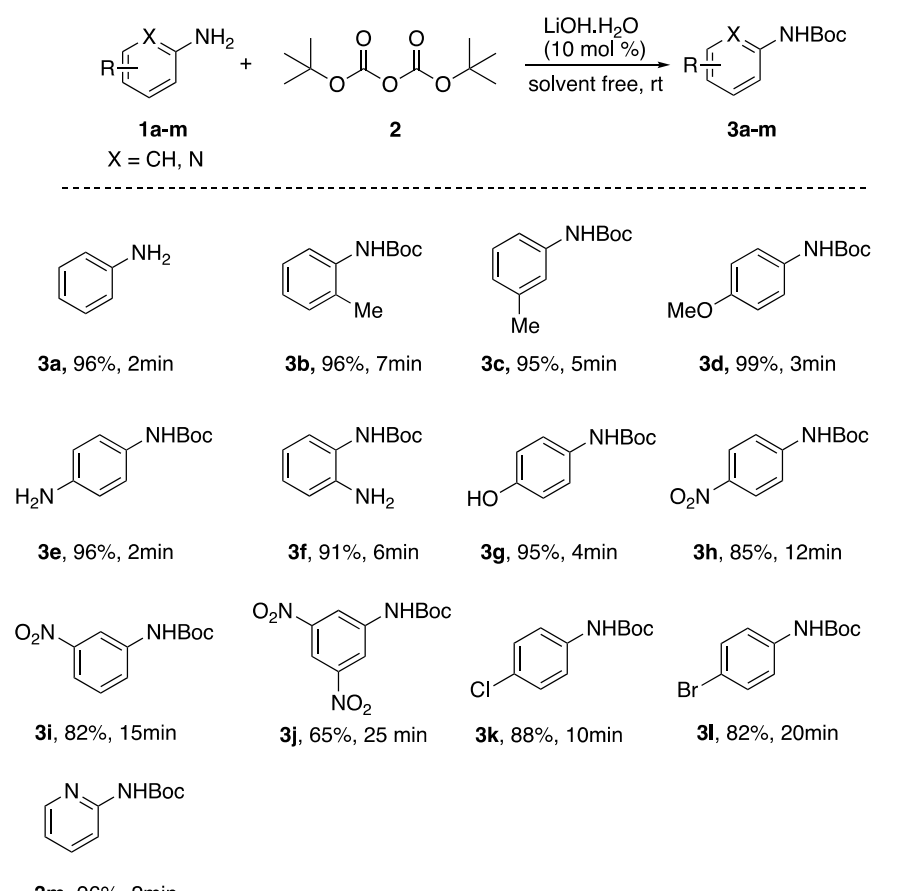

$3 \mathrm{~m}, 96 \%$, $2 \mathrm{~min}$

a Reaction conditions: 1a (1 mmol), 2 (1 mmol), LiOH. $\mathrm{H}_{2} \mathrm{O}(10 \mathrm{~mol} \%)$, in Solvent $(1 \mathrm{~mL})$ at room temperature for given time. 'Isolated.

Alkyl amines smoothly reacted to $(\mathrm{Boc})_{2} \mathrm{O}$ and afforded the corresponding exclusively mono-Boc protected products $3 \mathrm{n}-\mathrm{q}$ in the range of $89-91 \%$ yield. It is $100 \%$ mono-selective we did not observe diprotection of aliphatic amines. No comparative side reactions leading to formation of isocyanate, urea of $\mathrm{N}, \mathrm{N}-\mathrm{di}$-Boc derivatives were detected by thin layer chromatography, ${ }^{1} \mathrm{H}$ NMR and ${ }^{13} \mathrm{C}$ NMR analyses of the crude products.
Table 3. Substrate scope for N-Boc protection of aliphatic Aminesa, b

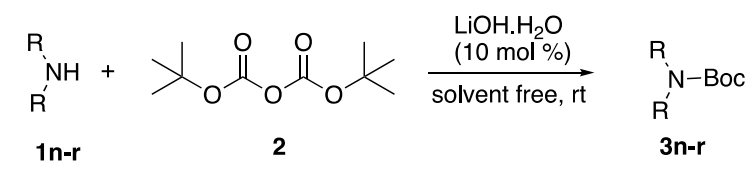

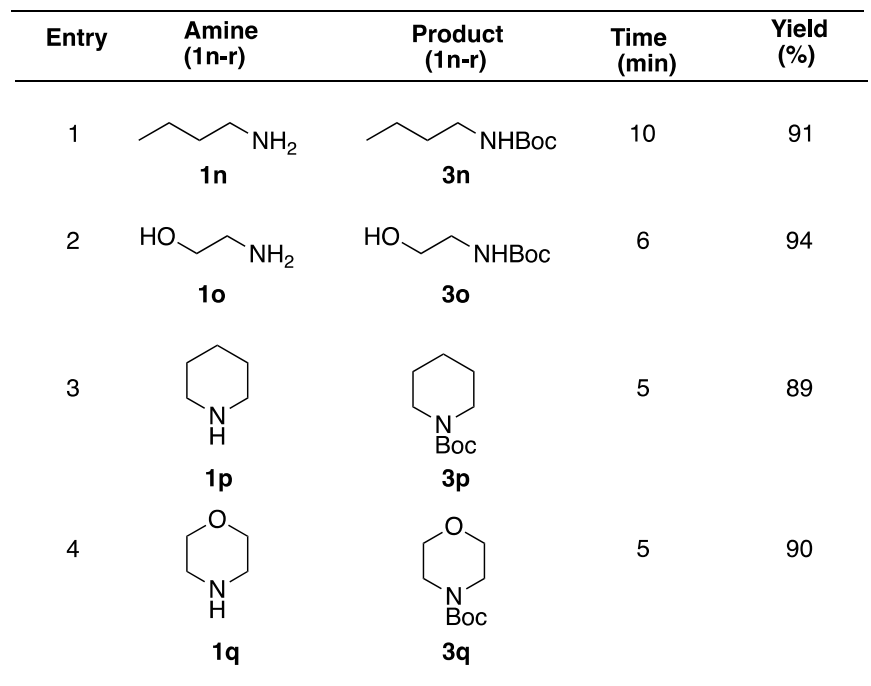

a Reaction conditions: 1a (1 mmol), 2 (1 mmol), $\mathrm{LiOH} . \mathrm{H}_{2} \mathrm{O}(10 \mathrm{~mol} \%)$, in Solvent $(1 \mathrm{~mL})$ at room temperature for given time. 'Isolated.

\section{CONCLUSION}

Thus we had designed a simple, rapid, efficient and green method for chemo-selective N-Boc protection of amines using Lithium hydroxide as a green catalyst in presence of ditert-butyl carbonate under solvent free condition at room temperature. In presences of electron withdrawing group as substituent's reaction occurs slowly while in presences of electron donating group reaction occurs rapidly. No competitive side reactions are observed in this protocol.

\section{ACKNOWLEDGMENTS}

The authors gratefully acknowledge the Central Instrumental Facility, Savitribai Phule, Pune University, Pune, India and DST - FIST sponsored Central Instrumentation Laboratory, Dada Patil 
Mahavidyalaya, Karjat Dist- Ahmednagar, India for the spectral analysis and financial support.

\section{Spectral data:}

3a Tert-butyl phenylcarbamate: White solid; m.p. $131-133^{\circ} \mathrm{C}$; Yield $96 \%$; ${ }^{1} \mathrm{H}$ NMR (400MHz, CDCl3, TMS, ppm): $\delta$ 7.367.26(m, $4 \mathrm{H}), 7.05-7.03(\mathrm{~m}, 1 \mathrm{H}), 6.46(\mathrm{bs}$, 1H), 1.52(s, 9H); ${ }^{13} \mathrm{C}$ NMR (100 MHz, CDCl3, ppm): $\delta 152.73,138.30,123.97$, 123.01, 118.49, 80.49, 28.33.

3b Tert-butyl o-tolylcarbamate : White solid; m.p.84-85 ${ }^{\circ} \mathrm{C}$; Yield 96\%; ${ }^{1} \mathrm{H}$ NMR (400 MHz, $\mathrm{CDCl}_{3}$, TMS, ppm): $\delta 7.83(\mathrm{~d}, \mathrm{~J}=$ $8 \mathrm{~Hz}, 1 \mathrm{H}), 7.22(\mathrm{t}, \mathrm{J}=8 \mathrm{~Hz}, 1 \mathrm{H}), 7.17(\mathrm{~d}, \mathrm{~J}=$ $8 \mathrm{~Hz}, 1 \mathrm{H}), 7.03(\mathrm{t}, \mathrm{J}=8 \mathrm{~Hz}, 1 \mathrm{H}), 6.37(\mathrm{bs}, 1 \mathrm{H})$, 2.28(s, 3H), 1.57(s, 9H); ${ }^{13} \mathrm{C}$ NMR (100 $\mathrm{MHz}, \mathrm{CDCl} 3$, ppm): $\delta$ 153.00, 136.20, $132.80,130.20,126.60,123.60,121.00$, $80.20,28.20,17.60$.

3c Tert-butyl m-tolylcarbamate: White solid; Yield 95\%; ${ }^{1} \mathrm{H}$ NMR (400 MHz, $\mathrm{CDCl} 3, \delta \mathrm{ppm}) 7.28(\mathrm{~s}, 1 \mathrm{H}), 7.18(\mathrm{~d}, \mathrm{~J}=$ $8 \mathrm{~Hz}, 1 \mathrm{H}), 7.13(\mathrm{~d}, \mathrm{~J}=8 \mathrm{~Hz}, 1 \mathrm{H}), 6.88(\mathrm{~d}, \mathrm{~J}=$ $8 \mathrm{~Hz}, 1 \mathrm{H}), 6.53(\mathrm{bs}, 1 \mathrm{H}), 2.35(\mathrm{~s}, 3 \mathrm{H}), 1.55(\mathrm{~s}$, 9H); ${ }^{13} \mathrm{C}$ NMR (100 MHz, CDCl3, ppm): $\delta$ 152.80, 138.80, 138.20, 128.70, 123.80, $119.10,115.60,80.30,28.30,21.40$.

\section{3d Tert-butyl (4-}

methoxyphenyl)carbamate : White solid; m.p. 94-96 ${ }^{\circ} \mathrm{C}$; Yield 99\%; ${ }^{1} \mathrm{H}$ NMR (400 $\mathrm{MHz}, \mathrm{CDCl} 3, \mathrm{ppm}): \delta 7.26(\mathrm{~d}, \mathrm{~J}=8 \mathrm{~Hz}, 2 \mathrm{H})$, $6.82(\mathrm{~d}, \mathrm{~J}=8 \mathrm{~Hz}, 2 \mathrm{H}), 6.44(\mathrm{bs}, 1 \mathrm{H}), 3.77$ (s, $3 \mathrm{H}), 1.50(\mathrm{~s}, 9 \mathrm{H}) ;{ }^{13} \mathrm{C} \mathrm{NMR}(100 \mathrm{MHz}$, $\mathrm{CDCl} 3, \mathrm{ppm}): \delta$ 155.60, 153.20, 131.40,120.50, 114.10, 80.10, 55.40, 28.30.

3e Tert-butyl (4-aminophenyl) carbamate: White solid; m.p.110-113 ${ }^{\circ} \mathrm{C}$; Yield 96\%; ${ }^{1} \mathrm{H}$ NMR
(400 MHz, CDCl3, TMS, ppm): $\delta 7.13(\mathrm{~d}, \mathrm{~J}=8 \mathrm{~Hz}, 2 \mathrm{H})$, $6.64(\mathrm{~d}, \mathrm{~J}=8 \mathrm{~Hz}, 2 \mathrm{H} 6.30$ (bs, $1 \mathrm{H}), 3.20$ (bs, 2H), 1.50(s, 9H); ${ }^{13} \mathrm{C}$ NMR (100 MHz, CDCl3, $\mathrm{ppm}): \delta 153.34,142.38,129.71,120.91$, $115.62,80.01,28.40$.

3f Tert-butyl (2-aminophenyl)carbamate :

White solid; m.p. $109-111^{\circ} \mathrm{C}$; Yield $91 \%$; ${ }^{1} \mathrm{H}$ NMR (400 MHz, CDCl3, TMS, ppm): $\delta$ $7.25(\mathrm{~d}, \mathrm{~J}=8 \mathrm{~Hz}, 1 \mathrm{H}), 7.01(\mathrm{~m}, 1 \mathrm{H})$, 6.80(m,2H), 6.22(bs, $1 \mathrm{H}), 3.72(\mathrm{bs}, 2 \mathrm{H})$, 1.51(s, 9H); ${ }^{13} \mathrm{C}$ NMR (100 $\mathrm{MHz}, \mathrm{CDCl}_{3}$, ppm): $\delta$ 153.94,139.18, 126.20, 125.09, 124.79, 120.13, 117.98, 80.67, 28.34 .

\section{3g Tert-butyl (4-}

hydroxyphenyl)carbamate : White solid; m.p. $145-147^{\circ} \mathrm{C}$; Yield 95\%; ${ }^{1} \mathrm{H}$ NMR (400 $\mathrm{MHz}, \mathrm{CDCl} 3$, TMS, ppm): $\delta 7.19(\mathrm{~d}, \mathrm{~J}=8 \mathrm{~Hz}$, $2 \mathrm{H}), 6.75(\mathrm{~d}, \mathrm{~J}=8 \mathrm{~Hz}, 2 \mathrm{H}), 6.40(\mathrm{bs}, 1 \mathrm{H})$, 4.90(bs, $1 \mathrm{H}), 1.51(\mathrm{~s}, 9 \mathrm{H}) ;{ }^{13} \mathrm{C}$ NMR (100 $\mathrm{MHz}, \mathrm{CDCl} 3, \mathrm{ppm}): \delta 153.63,152.12$, 130.84, 121.71, 115.75, 80.46, 28.38.

\section{3h Tert-butyl (4-nitrophenyl)carbamate :}

Yellowish Oil, b.p. $308-312^{\circ} \mathrm{C}$; Yield 85\%; ${ }^{1} \mathrm{H}$ NMR (300 MHz, CDCl3, TMS, ppm): $\delta$ 8.19-8.16(d, J =8Hz, 2H), 7.54-7.51(d, $\mathrm{J}=8 \mathrm{~Hz}, 2 \mathrm{H}), 6.89$ (bs, $1 \mathrm{H}), 1.47(\mathrm{~s}, 9 \mathrm{H}) ;{ }^{13} \mathrm{C}$ NMR (100 MHz, CDCl3, ppm): $\delta$ 152.70, 137.60, 126.30, 125.3, 113.00, 51.50, 29.10. 3i Tert-butyl (3-nitrophenyl)carbamate :

Yellow solid; m.p. $180-182{ }^{\circ} \mathrm{C}$; Yield 82\%; ${ }^{1} \mathrm{H}$ NMR (300 MHz, CDCl3, TMS, ppm): $\delta$ 8.30(s, $1 \mathrm{H}), 7.88(\mathrm{~d}, \mathrm{~J}=9 \mathrm{~Hz}, 1 \mathrm{H}), 7.68(\mathrm{~d}, \mathrm{~J}=$ $3 \mathrm{~Hz}, 1 \mathrm{H}), 7.44(\mathrm{t}, \mathrm{J}=9 \mathrm{~Hz}, 1 \mathrm{H}), 6.72(\mathrm{bs}, 1 \mathrm{H})$, 1.54(s, 8H); ${ }^{13} \mathrm{C}$ NMR (100 MHz, $\mathrm{CDCl} 3$, ppm): $\delta 152.40,149.20,139.90,129.90$, $124.10,117.80,113.40,81.80,28.50$.

\section{3k Tert-butyl (4-}

chlorophenyl)carbamate :White solid; m.p. 
$102-103^{\circ} \mathrm{C}$; $100-103^{\circ} \mathrm{C}$; Yield $88 \%{ }^{1} \mathrm{H}$ NMR (400 MHz, CDCl3, ppm): $\delta$ 7.29$7.32(\mathrm{~d}, \mathrm{~J}=9 \mathrm{~Hz}, 2 \mathrm{H}), 7.24(\mathrm{~d}, \mathrm{~J}=8 \mathrm{~Hz}, 2 \mathrm{H})$, 6.52(bs, 1H), 1.51(s, 9H); ${ }^{13} \mathrm{C}$ NMR (100 $\mathrm{MHz}, \mathrm{CDCl} 3, \mathrm{ppm}): \delta 151.80,144.40$, 142.70,125.10, 117.40, 81.90, 28.10.

\section{Tert-butyl (4-bromophenyl)carbamate :}

White solid; m.p.101-103 ${ }^{\circ} \mathrm{C}$; Yield $82 \%$; ${ }^{1} \mathrm{H}$ NMR (400 MHz, CDCl3, TMS, ppm): $\delta$ $7.39(\mathrm{~d}, \mathrm{~J}=8 \mathrm{~Hz}, 2 \mathrm{H}), 7.25(\mathrm{~d}, \mathrm{~J}=8 \mathrm{~Hz}, 2 \mathrm{H})$, 6.47(bs, 1H), 1.51(s, 9H); ${ }^{13} \mathrm{C}$ NMR (100 $\mathrm{MHz}, \mathrm{CDCl} 3, \mathrm{ppm}): \delta 152.50,137.46$, 131.89, 120.02,115.43, 80.92, 28.31.

\section{3m Tert-butyl pyridin-2-ylcarbamate :}

White solid; m.p.99- $101^{\circ} \mathrm{C}$; Yield 82\%; ${ }^{1} \mathrm{H}$ NMR (400 MHz, CDCl3, TMS, ppm): $\delta$ 11.23(s, $1 \mathrm{H}), 7.91(\mathrm{~d}, \mathrm{~J}=8 \mathrm{~Hz}, 1 \mathrm{H}), 7.81(\mathrm{~d}, \mathrm{~J}$ $=8 \mathrm{~Hz}, 1 \mathrm{H}), 7.39(\mathrm{~d}, \mathrm{~J}=8 \mathrm{~Hz}, 1 \mathrm{H}), 7.28(\mathrm{dd}, \mathrm{J}$ $=8 \& 4 \mathrm{~Hz}, 1 \mathrm{H}), 1.59(\mathrm{~s}, 9 \mathrm{H}) ;{ }^{13} \mathrm{C} \mathrm{NMR}$ (100 MHz, CDCl3, ppm): $\delta 161.37,152.85$, 152.62, 147.52, 138.34, 121.00, 80.87, 28.37 3n Tert-butyl butylcarbamate : Colorless oil; Yield 91\%; ${ }^{1} \mathrm{H}$ NMR (400 MHz, $\mathrm{CDCl} 3$, TMS, ppm): $\delta 4.49$ (bs, $1 \mathrm{H}), 3.10(\mathrm{t}, \mathrm{J}=8 \mathrm{~Hz}$, $2 \mathrm{H}), 1.48(\mathrm{~s}, 9 \mathrm{H}), 1.48-1.35(\mathrm{~m}, 2 \mathrm{H}), 1.34-$ 1.28(m, 2H), 0.91(t, J = 8Hz, 3H); ${ }^{13} \mathrm{C}$ NMR (100 MHz, CDCl3, ppm): $\delta 156.00$, 79.00, 40.40,32.20, 28.40, 19.90, 13.70.

\section{3o Tert-butyl (2-hydroxyethyl)carbamate :}

Colorless oil; b.p. $90-92^{\circ} \mathrm{C}$; Yield $94 \% ;{ }^{1} \mathrm{H}$ NMR (400 MHz, CDCl3, TMS, ppm): $\delta$ 5.23(bs, 1H), 3.66(t, 2H), 3.26(t, 2H), 2.75(s,1H), 1.43(s, 9H); ${ }^{13} \mathrm{C}$ NMR (100 $\mathrm{MHz}, \mathrm{CDCl} 3, \mathrm{ppm}): \delta 156.84,79.62,62.09$, 43.00, 28.35.

\section{3p Tert-butyl piperidine-1-carboxylate:}

Colorless oil; Yield 89\%; ${ }^{1}$ H NMR (400 MHz, d6-DMSO, TMS, ppm): $\delta$ 3.35(m,
4H), 1.56-1.45(m, 15H); ${ }^{13} \mathrm{C}$ NMR (100 $\mathrm{MHz}, \mathrm{d} 6-\mathrm{DMSO}, \mathrm{ppm}): \delta 154.89,79.03$, 44.31, 28.42, 25.69, 24.45.

\section{3q Tert-butyl morpholine-4-carboxylate} (Table 3, entry 10): White solid; m.p. 65$66^{\circ} \mathrm{C}$; Yield 90\%; ${ }^{1} \mathrm{H}$ NMR (400 MHz, CDCl3, TMS, ppm): $\delta 3.64(\mathrm{t}, \mathrm{J}=4 \mathrm{~Hz}, 4 \mathrm{H})$, $3.42(\mathrm{t}, \mathrm{J}=4 \mathrm{~Hz}, 4 \mathrm{H}), 1.47(\mathrm{~s}, 9 \mathrm{H}) ;{ }^{13} \mathrm{C} \mathrm{NMR}$ (100 MHz, CDCl3, ppm): $\delta 154.77,79.92$, $66.67,43.48,28.37$

\section{REFERENCES}

[1]. (a) T.W Greene.;P.G.M. Wuts. In Protecting group in organic synthesis; John Wiley and Sons: New York, (1999). (b) P. J. Kocienski. Protecting Group; George Thieme: New York, (2000).

[2]. G. Sartori, R. Ballin, F.Bigi, G. Bosica, R. Maggi, P. Right, Chemical Reviews. 104, 199, (2004).

[3]. Y. Basel, A. Hassner. J. Org. chem. 65, 6368, (2000).

[4]. E. Guibe-Jampel, M. Wakselman, Synthesis 65, 6368, (2000).

[5]. G. Barcelo, J.-PSenet; G.Sennyey, G.Synthesis 627-632, (1986).

[6]. M. Itoh, D. Hagiwara, T. Kamiya, Tetrahedron Lett, 4393-4394, (1975).

[7]. a) S. Darnbrough, M. Mervic, S. M. Condon and C. J. Burns, Synth. Commun. 31, 3273, (2001). b) H. J. Knolker, T. Braxmeier and G. Schlechtingen, Synlett, 502, (1996). c) H. J. Knolkerand T. Braxmeier TetrahedronLett. 37, 5861, (1996). d) H. J. Knolker, H. J. Braxmeier, T.Schlechtingen, G. Angew.Chem. Int. Ed. Engel. 34, 2497,(1995).

[8]. G. V. S, Sharma, J. J. Reddy, P. S. Lakshmi, P.R. Krishna. Tetrahedron Lett. 45, 69636965,(2004). 
[9]. A. Heydari, R. Kazemshiroodi, H. Hamadi, M. Esfandyari, M. Pour-ayoubi, Tetrahedron Lett. 48, 5865, (2007).

[10]. D. J. Upadhya, A. Barge, R. Stefenia, G. Cravotto,Tetrahedron Lett, 48, 8318, (2007).

[11]. K. C. Rajanna, Synth. Commun, 41,715, (2011).

[12]. R. Varala, S. Nuvula and S. R. Adapa, J.Orga.chem,71,8283, (2006).

[13]. S. Khaksar. A. Heydari, M. Tajbakhsh, S. M. Vahdat. TetrahedronLett, 49, 3527,(2008).

[14]. A. Hedari, S. E. Hosseini, Adv. Synth. Catal.347, 1929, (2005).

[15]. G. Bartoli, M. Bosco, M. Locatelli, E. Marcantoni, M. Massaccesi, P. Melchiorre, L. Shambri, Synlett10, 1794, (2004).

[16]. N. Suryakiran, P. Prabhakar, S. T. Reddy, S.T. Rajesh, K. Venkateswarlu, Tetrahedron Lett,47,8039-8042, (2006).

[17]. S. V. Chankeshwara and A. K. Chakraborti, Tetrahedron Lett, 47,1087, (2006).

[18]. A. K. Chakraborti, S. V. Chankeshwar, Org. Biomol. Chem.,4,2769-2771, (2006).

[19]. B. Das, K. Verkateswarlu, M. Krisnaiah, H. Holla, Tetrahedron Lett.47, 7551, (2006).

[20]. S. V. Chankeshwara and A. K. Chakraborti, Synthesis, 2784, (2006).

[21]. A. Heydari, S. Khaksar, M. Tajbakhsh, Synthesis, 19, 3126, (2008).

[22]. N. Inahashi, A. Matsumiya, and T. Sat.Synlett, 294, (2008).

[23]. S. V. Chankeshwara, A. K. Chakraborti, J. Mol. Catal. A.Chem, 253, 198, (2006).

[24]. A. Heydari, S. Khaksar and M. Tajbakhsh, Synthesis, 3126, (2008).

[25]. S. Khaksar, S. M. Vahdat, M. Tajbakhsh, F. Jahani and A. Heydari, Tetrahedron Lett, 51, 6388, (2010).

[26]. D. J. Upadyaya, A. Barge, R. Stetania and G.Cravotto, Tetrahedron Lett.48, 8313,(2007).

[27]. F. jahani, M. Tajbakhsh, H.Golchoubian and S. Khaksar, Tetrahedron Lett, 52, 1260,(2011).
[28]. B. Das, K. Venkateswarlu, M. Krishnaiah, H. Holla, Tetrahedron Lett.47, 7571-7556, (2006).

[29]. R. K. Pandey, S. P. Dagad, R. K. Upadhayay, M. K. Dongare, P. Kumara, ARKIVOC, vii, 28-33, (2002).

[30]. M. S. Reddy, M. Narender, Y. V. D. Nageswarand K. R. Rao, Synlett, 1110, (2006).

[31]. S. V. Chankeshwara and A. K. Chakraborti, Org. Lett, 8,3259,(2006).

[32]. T. Vilaivan, Tetrahedron Lett, 47, 6739, (2006).

[33]. a) V. Siddaiah, G. M. Basha, G. P. Rao, U. V. Pasad and R. S. Rao,Chem, Let,39, 1127, (2010). b) H. Zeng, Y. Li and H. Shao, Synt.Commun, 42, 25, (2012).

[34]. a) X. Jia, Q. Huang, J. Li, S. Li and Q Yang, Synlett,0806, (2007). b) S. N. Dighe and H. R. Jadhav, H.R. Tetrahedron Lett.53, 5803, (2012). c) M. Nardi, N. H. Cano, P. Costanzo, M. Oliverio, G. Sindon and Procopio, A. RSCAdv, 5, 18751,(2015).

[35]. A. Ingale, S. V. Shide, V. K. More, U. S. Gangarde, New J. Chem., DOI: 10.1039/ C8NJ01585F (2018).

[36]. J. Dharita, Upadhya, Alessandro Barge, Rachele Stefania, and Giancarlo Cravotto, 48, 83188322,(2007).

[37]. JafarAkabar, Akbar Heydari, Leila Mamani, SeyedHassa Hosseini,13,544-547(2010).

\section{Cite this article as :}

Sandip P. Gondake, Santosh R. Kshirsagar, Ashok S. Pise, Valmik S. Kapase, Sagar I. Shinde, "Green approach to Chemo-Selective N-Boc Protection of Amines using Catalytic amount of Lithium Hydroxide Monohydrate under Solvent Free Condition", International Journal of Scientific Research in Science and Technology (IJSRST), Online ISSN : 2395-602X, Print ISSN : 2395-6011, Volume 7 Issue 5, pp. 06-12, September-October 2020. Available at doi : https://doi.org/10.32628/IJSRST20751 Journal URL : http://ijsrst.com/IJSRST20751 